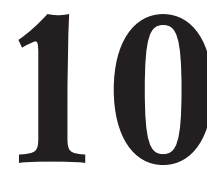

\title{
FALANGE Y EDUCACIÓN: EL SEPEM Y EL DEBATE SOBRE EL BACHILLERATO EN LOS AÑOS CUARENTA
}

\author{
(FALANGE AND EDUCATION: THE SEPEM AND THE DEBATE ON \\ SECONDARY EDUCATION DURING THE FORTIES)
}

Antonio Fco. Canales Serrano

Universidad de La Laguna

\section{RESUMEN}

Este artículo estudia los planteamientos y la actuación falangista en la enseñanza media durante el primer franquismo. La principal plataforma en este ámbito para los falangistas fue el Servicio Español de Profesorado de Enseñanza Media, integrado en la Delegación Nacional de Educación del Partido, que aspiraba a encuadrar al profesorado de enseñanza media, tanto oficial como privado. A lo largo de los años cuarenta, el SEPEM lideró la movilización falangista contra el modelo de bachillerato establecido en 1938 en abierta oposición a los sectores católicos. El artículo analiza la organización del SEPEM, sus planteamientos sobre el bachillerato y las infructuosas campañas en favor de la reforma que impulsó. Finalmente, plantea la debilidad estructural del Servicio y su incapacidad para llevar a cabo su cometido de encuadramiento y movilización del profesorado.

\section{ABSTRACT}

This article studies the positions and activities of the Falange in secondary education during the first Francoism. The main falangist platform in this area was the Servicio Español del Profesorado de Enseñanza Media, integrated in the Delegación Nacional de Educación of the Party, which aspired to control secondary education teachers, both official and private. During the forties, the SEPEM led the falangist mobilization against the model of secondary education established in 1938 in open opposition to the Catholic sectors. The article 
analyzes the organization of the SEPEM, its positions on secondary education and the fruitless campaigns that it promoted in favour of reform. Finally, it outlines the structural weakness of the Service and its failure controlling and mobilizing teachers.

\section{INTRODUCCIÓN}

La preeminencia católica en la educación española de posguerra parece difícil de cuestionar. Es ampliamente aceptado entre los especialistas que, en el reparto de áreas de influencia entre los diversos grupos que apoyaban al régimen franquista, la educación correspondió al sector católico (Ruiz Rico, 1977). Los católicos tuvieron una destacada presencia ya en ya en la Comisión de Cultura y Enseñanza de octubre de 1936 y la mantuvieron en el posterior Ministerio de Educación Nacional (Cámara, 1984, Alted, 1984). De hecho, lograron una victoria decisiva en la enseñanza media al establecer un modelo de bachillerato que satisfacía sus reivindicaciones tradicionales, incluso antes de que concluyese la guerra. Resulta congruente, pues, que buena parte de la historiografía sobre la educación en los años cuarenta, especialmente sobre la enseñanza media, se haya centrado en el estudio de la actuación de la Iglesia, agente clave para comprender la realidad educativa del periodo (Alfonso, 1999).

Ahora bien, este predominio del proyecto educativo católico no justifica la escasa atención prestada por la historiografía educativa a la Falange, el otro componente fundamental del régimen. A pesar de que se postula genéricamente una tensión permanente entre la Iglesia y la Falange, la actuación falangista sólo ha sido objeto de estudio detallado en dos esferas educativas: la universitaria, en la que es común hacer referencia a los falangistas y al SEU (Ruiz Carnicer, 1996), y el encuadramiento de la juventud a través del Frente de Juventudes (Alcoba, 2002; Cruz, 2001; Sáenz, 1988) y la Sección Femenina (Noval, 1999). En el resto de los ámbitos educativos de posguerra, el falangismo permanece emboscado en las sombras, a la manera de un fantasmagórico grupo de presión que irrumpe esporádicamente en el debate educativo para consternación de la Jerarquía católica, y cuyos planteamientos, objetivos y estrategias se infieren por oposición más que estudiarse en sí mismos.

Los falangistas de posguerra, sin embargo, distaban bastante de actuar en la sombra. Por el contrario, la Falange impulsó diversos organismos y organizaciones para intervenir abiertamente en el mundo educativo. Además del SEU, el Frente de Juventudes y la Sección Femenina, se creó dentro del Partido un organismo centrado específicamente en el sistema educativo 
formal, la Delegación Nacional de Educación, y en su seno se desarrollaron organizaciones destinadas a encuadrar al profesorado e influir decisivamente en las características de la nueva educación, como ya mostró hace años González Gallego (1988) para el caso zaragozano, sin que la línea abierta por este investigador haya tenido continuidad. Posiblemente, el más conocido de estos organismos sea el Servicio Español del Magisterio (SEM), que, aunque no ha sido objeto de ningún estudio monográfico, constituye una referencia obligada dentro de los estudios sobre el Magisterio. Pero funcionaron también el Servicio Español del Profesorado de Enseñanza Superior (SEPES), Técnica (SEPET) y Media (SEPEM), todos ellos prácticamente desconocidos.

Este artículo constituye una primera aproximación al SEPEM tras el rastreo de las escasas y dispersas fuentes que se conservan en el Archivo General de la Administración. A partir de este material y de publicaciones de la época, el artículo estudia los planteamientos falangistas sobre el bachillerato y la amplia movilización del profesorado que la Falange impulsó a través del SEPEM en favor de la reforma del bachillerato establecido en 1938. Finalmente, evalúa la debilidad estructural de este instrumento diseñado para el encuadramiento del profesorado y las razones de su práctica desaparición tras las sucesivas derrotas en las campañas que impulsó.

Rescatar al SEPEM del olvido historiográfico responde a una doble pretensión. De un lado, contribuir a la caracterización de uno de los contendientes fundamentales en la pugna educativa de posguerra. De otro, llamar la atención sobre la influencia fascista en la educación española de los años cuarenta en el sentido propuesto por Morente (2005).

\section{LA PRIMERA ACTIVIDAD DEL SEPEM}

Desde la Unificación existían varios organismos en el partido centrados en temas educativos, entre los que destacaban el Sindicato Español Universitario (SEU) y el Servicio Español del Magisterio (SEM). En 1940 estos organismos se reorganizaron en el Servicio Nacional de Educación, denominado posteriormente Delegación Nacional de Educación Nacional. En su seno se crearon los servicios del profesorado de enseñanza superior (SEPES), media (SEPEM) y técnica (SEPET), además del ya existente SEM, con la pretensión de encuadrar a todos los profesionales de la docencia de cada uno de los tramos educativos en una amplia corporación profesionali.

La Delegación se arrogaba la iniciativa, la asesoría y el control de la política educativa con el fin de garantizar la vigencia de los principios na- 
cional-sindicalistas en la educación de posguerra y ser el cauce de expresión del ideario educativo falangista. No obstante, en el ámbito de la enseñanza media la actividad de la Delegación resultó extremadamente limitada en la inmediata posguerra con la excepción del núcleo falangista de Zaragoza que editaba desde la guerra la combativa revista Educación y Cultura. No es extraño, pues, que, en contraste con la atonía generalizada, el SEPEM diera pronto cuenta de su voluntad intervencionista en ese distrito. En 1941 el SEPEM zaragozano había conseguido negociar con los representantes de la enseñanza privada las condiciones de trabajo de los licenciados contratados y, tras algunas tensiones con el Ministerio, que se reconociera su derecho a ingresar el 2 por mil de la masa salarial y a obtener una copia de todos los contratos firmados. ${ }^{\text {ii }}$ A pesar de este precedente, el SEPEM no comenzó a actuar de manera significativa a escala nacional hasta 1942.

El primer indicio de que el falangismo abandonaba la atonía que había caracterizado su actuación hasta el momento en el ámbito de la enseñanza media fue la publicación en la primavera de 1942 de la revista Información Universitaria. ${ }^{\text {iii }}$ La nueva revista se hacía eco de la permanente campaña contra la ley de 1938 de la revista zaragozana Educación y Cultura. En mayo publicaba las conclusiones de una encuesta que defendían la supresión del examen de Estado, una de las novedades claves del bachillerato franquista, y la exclusividad de los institutos para cualquier tipo de prueba, «atendiendo al carácter estatal que impera en todos los órdenes del Estado nacionalsindicalista». ${ }^{\text {iv }}$ Nuevas críticas hacia el examen de Estado se publicaron tras la celebración del correspondiente al curso en el que, según la revista, sólo el 30\% de los presentados aprobó en Madrid. En esta ocasión la línea argumentativa contraponía las buenas intenciones del examen a la nocividad de sus efectos. La libertad concedida a todo tipo de preparadores externos a la enseñanza oficial habría provocado en pocos años la aparición de una generación de revalistas con conocimientos meramente memorísticos que nada tenía que ver con los objetivos formativos que el examen pretendía garantizar.

No tienen ellos la culpa, pero sí el sistema que, dejando al arbitrio de colegios empresas, licenciados particulares, padres de los propios alumnos, etcétera, ha producido al cabo de unas cuentas convocatorias el examinando «estandarizado» que sabe repetir en contados minutos la definición del átomo, las obras de Calderón y hasta los ríos de Ceilán.

La solución pasaba por el retorno del examen a los institutos, «cada cual a su oficio», medida que además "supondría - digámoslo con claridad- poner al profesorado oficial de nuestros centros de Enseñanza Media en el puesto que por derecho propio les corresponde, y de paso se 'jerarqui- 
zaría' la función docente de la enseñanza. Y en un Estado fuerte la enseñanza debe tener consideración preeminente».v

El nuevo director general de enseñanza media Luis Ortiz Muñoz, quien sustituyó a José Pemartín en julio de 1942, abrió nuevas posibilidades de actuación al Servicio. Una de las primeras actuaciones de Ortiz, quien compaginó su nuevo cargo con la secretaría del SEPEM, fue anunciar la convocatoria de una Semana de la Enseñanza Media Oficial que había de abordar los problemas del sector. ${ }^{\text {vi }}$ Se trataba de una iniciativa muy significativa, pues la semana suponía un contrapeso importante a las Semanas de Educación Nacional que la FAE celebraba anualmente y cuyas conclusiones encontraban amplio eco en la prensa católica y las publicaciones educativas. La convocatoria de Ortiz rompía el monopolio católico de los foros de reflexión educativa característico del mandato de Pemartín.

Paralelamente, el Ministerio creaba un nuevo organismo al que dotaba de importantes y significativas competencias: la Comisión Consultiva de Enseñanza Media. Su pretendido carácter técnico-administrativo permitía eludir la inclusión de representantes de las órdenes y congregaciones docentes o jerarquías eclesiásticas, presentes en todos los organismos educativos del momento, y establecer la hegemonía de la enseñanza pública entre sus miembros. Las competencias que se conferían al nuevo organismo tenían, sin embargo, poco de técnicas. Más allá de la asesoría, la Comisión se erigía en una especie de órgano deliberativo con capacidad para discutir mociones presentadas por los institutos y dictaminar sobre cualquier cuestión planteada por sus miembros, todo ello acompañado de la facultad de solicitar resoluciones sobre los asuntos que estimara convenientes a la Dirección General. Tanto sus competencias como su composición parecen apuntar a que la Comisión Consultiva podía ser la fórmula para llevar a cabo efectivamente en la enseñanza media las funciones de asesoría e iniciativa reconocidas a la Delegación Nacional de Educación del Partido. En todo caso, si llegaba a funcionar, era un órgano de extenso poder sobre las políticas de la Dirección General exclusivamente en manos de los representantes de la enseñanza oficial.

A finales de 1942 se celebró en Madrid la anunciada Primera Semana de Enseñanza Media Oficial, ${ }^{\text {viii }}$ que supuso un amplio esfuerzo de movilización del cuerpo de catedráticos. Asistieron a los actos 232 catedráticos, de ellos 57 directores de instituto. La semana confirmaba la fulgurante ascensión de José Navarro Latorre, adscrito poco antes al Ministerio y a los servicios centrales del SEPEM e incluido en la Comisión Consultiva de Enseñanza Media. José Navarro había sido hasta su traslado a Madrid delegado provincial de educación nacional en Zaragoza (González 1988, 203) y había 
participado como organizador del Servicio del Profesorado ya en 1936. ${ }^{\text {ix }}$ Era, por tanto, un auténtico camisa vieja del falangismo educativo que en febrero de 1943 se hacía con la vicesecretaría del SEPEM. Poco después, en mayo de 1943, el SEPEM celebraba su I Consejo Nacional en el que definía su postura sobre los temas claves del bachillerato de posguerra.

A lo largo de 1943 y 1944 el SEPEM puso en práctica una nueva estrategia que aspiraba a incrementar su control sobre el profesorado de los colegios privados: la incorporación al Servicio de los Colegios Oficiales de Licenciados y Doctores. El control de los Colegios Oficiales introducía una segunda línea de ataque falangista a la situación creada en la enseñanza media. A los principios generales y a la defensa de las competencias de los catedráticos de instituto, se añadía ahora la defensa genérica de los licenciados, lo que ponía sobre el tapete no sólo sus condiciones laborales, sino también su espacio en el mercado laboral. Todo ello remitía al incumplimiento por parte de los colegios privados de los requisitos de titulación de su profesorado, práctica sobre la que se acumulaban denuncias en la inspección del Ministerio. En este sentido, un informe del Colegio Oficial de Licenciados y Doctores de Madrid mantenía en 1943 que sólo el 3.65\% de los centros reconocidos del distrito universitario funcionaba plenamente según la ley. ${ }^{\mathrm{x}}$

\section{LOS PLANTEAMIENTOS SOBRE LA ENSEÑANZA MEDIA DEL SEPEM}

A principios de 1945, tras haber consolidado sus instrumentos de control sobre el profesorado tanto oficial como privado, el SEPEM estaba en condiciones de retornar al debate público sobre la reforma de la enseñanza media con la publicación en enero de los acuerdos de su Comisión Permanente, al parecer de noviembre de $1944 .{ }^{\mathrm{xi}}$

Los acuerdos desarrollaban las conclusiones del Consejo Nacional de 1943 y suponían una revisión en profundidad de los principios que vertebraban la Ley de 1938. Frente al sistema cíclico, que se consideraba que producía el recargo de asignaturas, se defendía la introducción de ciclos reducidos de materias o un sistema gradual, propuesta que parecía apuntar a la restauración del oficialmente denostado enciclopedismo anterior a la guerra. Esta crítica al sistema cíclico conducía directamente a la de su principal consecuencia: la supresión de exámenes intermedios para evitar una preparación meramente memorística. En este punto, el SEPEM criticaba las contradicciones de la Ley de 1938 y ponía de manifiesto que, en realidad, lo único que se había suprimido eran los exámenes oficiales en los institutos, 
no los exámenes anuales por asignaturas que la misma ley reconocía como método usual de evaluación. Además, subrayaba la contradicción existente entre la supresión de estos exámenes y la introducción de un examen general final: «Quede, pues, patente cómo no es posible rechazar el examen intermedio para evitar el memorismo y mantener el de Estado contra esta razón; si se admite uno el otro debe admitirse también».

Todo ello remitía a la crítica radical de la separación entre la función docente y la evaluadora que vertebraba el modelo vigente. A juicio del SEPEM, tras este principio subyacía la desconfianza ante la capacidad del profesorado para juzgar su propia obra. Sin embargo, esta idea no sólo quedaba refutada por la práctica, sino que resultaba contradictoria con el método establecido por la Ley para pasar de curso. "No hay razones fundamentales para que los profesores puedan calificar en los cursos del bachillerato, tanto los de enseñanza oficial como privada, y no puedan calificar la prueba final los primeros». Como pone de manifiesto el redactado de esta última frase, el argumento fundamental del SEPEM no era la defensa de la capacidad de los profesores en general para evaluar, sino la exclusividad del profesorado de instituto para juzgar aquellas pruebas que daban lugar a un título oficial. En este sentido, los dirigentes del SEPEM iban más allá de la crítica habitual a la falta de licenciados entre el profesorado de los colegios. No se trataba ya de una cuestión de titulación, sino de cuestionar abiertamente la equiparación entre profesorado oficial y privado que la ley planteaba, «cuando unas oposiciones establecen profunda disparidad para el ingreso respectivo en la docencia». En conclusión, «debe anularse el principio separador de funciones, no porque la experiencia haya demostrado sus ventajas e inconvenientes, sino porque encierra en sí una injusticia».

Tras estas críticas, el SEPEM esbozaba las líneas básicas de su propuesta de reforma. Aunque se reconocía su función preparatoria para la prosecución de otros estudios, se consideraba a la enseñanza media "como un ciclo educativo con sustantividad propia que proporciona una formación de tipo general completa». El nuevo bachillerato propuesto por el SEPEM tendría seis cursos, y no siete, y se dividiría en elemental de tipo único y superior con tres modalidades: realista, clásica y especial, adaptada esta última a las realidades regionales. Todos ellas darían acceso a la Universidad. Se cuestionaba así la hegemonía de las humanidades clásicas, otro de los pilares del bachillerato de 1938. Sin embargo, a pesar de la declaración inicial de sustantividad propia, la propuesta seguía considerando al bachillerato como esencialmente universitario. En realidad, suponía una reorganización mucho menos coherente y ambiciosa que el fracasado intento de José Pemartín de 1941 de coordinar las enseñanzas medias, pues obviaba el problema principal al que Pemartín había intentado dar respuesta: la integración en una úni- 
ca estructura de todos los estudios de enseñanza media y la creación de estudios medios con salida profesional. Se desentendía, además, el SEPEM del debate sobre un hipotético bachillerato femenino: «el problema de los Institutos femeninos será resuelto por el Estado conforme a sus posibilidades y circunstancias locales». De hecho, la propuesta del SEPEM se limitaba a reintroducir el bachillerato elemental y a abrir el debate sobre la especialización del superior.

El corto alcance de la propuesta de reforma del SEPEM era fruto de la cuestión de fondo que lastraba todo su programa: la defensa de las competencias reguladoras de los institutos sobre todo el sistema y, en consecuencia, de sus catedráticos, reparando la injusticia cometida en 1938. Así, frente al carácter romo y vago del diseño estructural propuesto para el bachillerato, los acuerdos se extendían larga y detalladamente en la regulación de cuestiones aparentemente burocráticas como matrícula, exámenes y centros. El principio de partida era que los institutos «tendrán una doble función: docente y reguladora», mientras los colegios sólo docente. De ahí se derivaba que todas las pruebas se celebrarían en los institutos, desde el examen de ingreso hasta la final, pasando por la prueba intermedia del bachillerato elemental. Se defendía, además, la clasificación de los colegios en cuatro categorías: reconocidos, incorporados, menores y libres. Los alumnos de los primeros se someterían sólo a las pruebas intermedia y final, mientras que los del resto deberían examinarse anualmente. En definitiva, lejos de hacerse eco del debate europeo sobre la reforma de la enseñanza media, siquiera en su versión fascista o nazi, las propuestas falangistas apenas se despegaban de las reivindicaciones corporativas de los catedráticos de instituto.

Un mes después, en febrero de 1945, Información Universitaria reproducía el mensaje que Fernando Solano Costa, delegado de educación en Zaragoza, leyó al ministro Ibáñez dando cuenta de estos acuerdos de la Comisión Permanente. ${ }^{\text {xii }}$ El mensaje calificaba a la Ley de 1938 de «absolutamente ineficaz y nociva para la formación de la juventud española» y responsable de la instauración de un sistema que "ha tornado el noble ejercicio de la docencia en una ocupación profesional sin más objetivo que el apetito de bienes materiales [...] Ley insostenible, su excesiva prolongación fomenta la depauperación intelectual y aun física del mejor de los tesoros de España: su juventud». Tras este radical diagnóstico, el mensaje señalaba abiertamente a los católicos como responsables de la situación y subrayaba su postura contradictoria. El SEPEM acataba la verdadera doctrina pontificia, pero se oponía a «que grupo de señores monopolice la ortodoxia, maneje un arma de dos filos - la intangibilidad de la Ley y su no aplicación». A su juicio, la intransigencia católica ponía en peligro sus propios intereses, pues «la enseñanza privada, la cristiana libertad de enseñanza, los fueros de una auténtica formación de la 
adolescencia española, se hallan amenazados por esa ley pendular de las pasiones contrarias que no habría de necesitar un cambio político para proyectarse como reacción al actual estado de cosas». ${ }^{\mathrm{xii}}$ Ante este peligro, los redactores del mensaje, "cristianos y falangistas», se presentaban como el justo término medio que cifraba la solución en una «nueva ley purgada de toda visión pequeña, utilitaria o sectaria». Ahora bien, este "camino de puro idealismo que busca una auténtica formación cristiana y nacional» que el SEPEM encarnaba necesitaba de libertad para combatir a los responsables de la situación. «Por ello, excelentísimo señor, os pedimos disciplinadamente una meditada libertad de expresión que contrarreste los efectos de esa desorbitada información del núcleo al que hemos aludido». A través del mensaje, en definitiva, el SEPEM señalaba al lobby católico como principal responsable de una situación a la que se oponía sin paliativos y mostraba su disposición a combatirlo dentro de los márgenes del debate político establecidos por el régimen.

\section{EL FRACASO DE LAS MOVILIZACIONES POR LA REFORMA}

A partir de 1946 el SEPEM impulsó una amplia movilización en favor de la reforma del bachillerato que alcanzaría su clímax en la discusión del Anteproyecto de Ley de Enseñanza Media de 1947. Esta movilización suponía importantes novedades con respecto a la actividad desplegada hasta el momento. No sólo se trataba de que las propuestas del SEPEM se expresaran públicamente con claridad y con abierta contundencia, sino que además se completó la campaña de prensa con la movilización de los claustros de los institutos que daría lugar a una sucesión de declaraciones colectivas. La campaña culminaría en 1947 con la presentación al cardenal Pla i Deniel de un escrito firmado por más de seis mil profesores, según fuentes falangistas.

En mayo de 1946, una Asamblea Nacional de Graduados celebrada en Madrid suscribía los puntos básicos de la propuesta del SEPEM, añadiendo la exclusividad de licenciados en centros privados y la revisión de las bases de trabajo. ${ }^{\text {xiii }}$ En otoño se iniciaba la publicación en Información Universitaria de escritos colectivos surgidos de los claustros de los institutos. Al escrito del profesorado del instituto Padre Suárez de Granada le sucedían en cascada los del profesorado de Valladolid, Vitoria y Pamplona, de los directores de instituto de Barcelona, Madrid y Galicia y, finalmente, de la casi totalidad de directores. ${ }^{\text {xiv }}$ La movilización no se limitaba a los profesionales de la enseñanza media oficial, sino que incluía también pronunciamientos del profesorado universitario en contra también del examen de Estado.

Los diferentes escritos, además de reclamar una reforma y el retorno 
de las competencias examinadoras a los institutos, dirigían abiertamente sus críticas a los sectores católicos a través de dos líneas argumentativas básicas. La primera situaba en primer plano los beneficios materiales que los colegios religiosos obtenían de la situación de facto de ausencia de control. En este sentido, el catedrático del instituto Menéndez Pelayo de Barcelona, José M. Gallard, denunciaba las dos estrategias más comunes de los colegios: «una admitir muchos alumnos en los primeros cursos y luego seleccionar al final. Dos suprimir todas las disciplinas que no van a examen, lenguas vivas, dibujo, griego, matemáticas...». ${ }^{\mathrm{xv}}$ La segunda línea de crítica, consecuencia de la primera, consistía en descalificar los argumentos católicos, especialmente los de la FAE, como propios de una parte interesada, tal y como denunciaban los profesores de Granada. ${ }^{x v i}$ Pero, sin duda, la diatriba más certera contra la estrategia católica de vincular la regulación del bachillerato con Franco fuera la de los profesores de Valladolid: «Es realmente ridículo pretender que la Cruzada Nacional se hizo por el Examen de Estado».xvii

La campaña tuvo una amplia repercusión en la prensa, tanto de provincias como de la capital. Las intervenciones de la FAE o de otros defensores del examen de Estado daban lugar a réplicas y contrarréplicas que amplificaban el debate. Tras la movilización se encontraba José Navarro, que había sido ascendido desde la vicesecretaría del SEPEM a secretario de la Delegación Nacional de Educación en enero de 1945. Un año después compaginaría este cargo con el de procurador en Cortes. Su mano derecha al frente del SEPEM era Antonio Fernández Pacheco, quien desplegó una intensa actividad para implicar a la estructura provincial y de distrito de la Delegación en la campaña. Suya era, por ejemplo, la consigna de que la Ley de

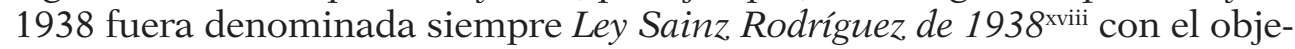
tivo de contrarrestar la estrategia católica que hacía referencia a ella como la ley de Franco y a la vez recordar que su responsable era una persona que había tenido que exiliarse a Portugal.

La campaña del SEPEM acompañó la tormentosa discusión en el Consejo Nacional de Educación del anteproyecto ministerial de reforma del bachillerato que se enfrentó a una dura oposición de los representantes del mundo educativo católico. Las dificultades que estaba sufriendo su tramitación llevaron al SEPEM a quemar su último cartucho. En octubre de 1947, poco antes de que el ya desvirtuado anteproyecto fuera discutido por la Comisión Permanente del Consejo de Educación Nacional, el SEPEM envió un manifiesto firmado, según los falangistas, por seis mil profesores y otras personas relacionadas con la cultura al Cardenal Primado Pla y Deniel, al nuncio y a todos los obispos. ${ }^{\text {xix }}$ 
El manifiesto comenzaba con una crítica implícita a la actitud de la jerarquía eclesiástica en el ámbito educativo y una advertencia de los peligros que de ella se derivaban. «La Jerarquía eclesiástica no puede representar en esta polémica los intereses particulares de la Enseñanza de los colegios regidos por Comunidades religiosas, y menos los de la enseñanza media seglar», pues ello socavaría su autoridad para desempeñar la dirección religiosa del conjunto del sistema educativo. «Por tanto, mantener posiciones dialécticas irreductibles y buscar que la autoridad de la Iglesia tome parte en pugnas que pueden enmascarar desviados propósitos, ofrece a nuestro entender, incalculable riesgo».

Tras esta advertencia, el escrito se articulaba en diez puntos, todos ellos defendidos con múltiples citas del derecho canónico, encíclicas y demás referencias que utilizaban habitualmente los defensores de la enseñanza religiosa. En el primero los firmantes declaraban con rotundidad su oposición al monopolio escolar estatal, tanto directo como indirecto. De ahí, el reconocimiento del derecho de la Iglesia a poseer sus propios centros. Ahora bien, la defensa del bien común confería al Estado el derecho y el deber de intervenir en la vida docente y, más aún, de fundar sus propios centros de enseñanza. La doble red, pública y privada, reconocida en la Ley de 1938 no era objeto de crítica, pero la situación vigente requería de una intensificación de la acción inspectora del Estado. En este punto se diferenciaba entre los colegios solventes tradicionales, a los que la Ley de 1938 «no ha menoscabado, sino que ha realzado en su crédito», y los nuevos colegios que "se han lanzado a enseñar bachillerato con rápida improvisación de medios técnicos y personales». Ante su escasa calidad el Estado no debía permanecer indiferente.

A continuación se defendían las pruebas estatales como la mejor garantía de la calidad. En este apartado se criticaba el examen de Estado por ser un «remedio tardío» y se abogaba por exámenes más frecuentes «a la manera de un reconocimiento médico, que comprueba periódicamente el estado físico y orienta sobre los medios para restaurar o conservar su normalidad». El siguiente paso de la argumentación era reclamar para el profesorado oficial la competencia para realizar estos exámenes. Llegados a este punto, el manifiesto se extendía en «reivindicar al cuerpo de catedráticos de enseñanza media, injustamente atacados».

El punto noveno tenía como objetivo conjurar las críticas católicas a estos exámenes oficiales afirmando que su existencia no conducía al monopolio estatal indirecto. En primer lugar, porque los alumnos de la enseñanza oficial también se sometían a pruebas. En segundo, y aquí la argumentación devenía especialmente insidiosa, porque la elección del centro había de depender de otras cuestiones mucho más importantes que la voluntad de eludir un examen: 
Y si, contra ésta nuestra opinión, el «deseo de no examinarse» determinase a un estudiante a cambiar de centro o influyera decisivamente en la elección, formaríamos lógicamente un triste concepto, tanto de la condición escolar del alumno y de su confianza en el propio trabajo y valer, como del ascendiente moral, del predicamento, de los alicientes y atractivos de orden elevado que no pudo hacer prevalecer contra esa menguada y aparente ventaja, el colegio preterido.

Finalmente, el punto décimo proponía una gradación de las pruebas oficiales según el tipo de centro similar a la defendida por el SEPEM. Los alumnos de colegios de reconocida solvencia sólo se someterían a examen para la obtención de un grado refrendado en título oficial, los de los colegios de menor categoría con una frecuencia mayor, pero a concretar, y los alumnos libres en todos los cursos y asignaturas.

Todas estas cuestiones fueron expuestas personalmente por el catedrático de Zaragoza, Miguel Allué Salvador, al Cardenal Primado, quien se vió forzado a recibirle. Sin embargo, esta estrategia no hacía más que reforzar la autoridad de la Iglesia y revelaba que, tal como iban las cosas, al SEPEM poco más le quedaba que apelar a la jerarquía eclesiástica. Y la Iglesia no estaba dispuesta a considerar ninguna alteración del modelo de 1938 como quedó de manifiesto cuando el Ministerio abandonó la tramitación el anteproyecto.

El fracaso palmario de la ofensiva reformista de 1946-47 revelaba que el SEPEM era incapaz de ofrecer satisfacción a las reivindicaciones del amplio frente que había movilizado. El sentido y la credibilidad del Servicio habían de salir seriamente cuestionados de la estéril campaña. Y efectivamente, su actividad se redujo notablemente en los años siguientes. No obstante, los dirigentes del Servicio no cejaban en su empeño de conseguir una reforma general de la enseñanza media. En palabras de Navarro, el SEPEM «no renuncia a la bandera, que antes que nadie tremoló», pues quedaba pendiente la "gran batalla de la Enseñanza Media española».xx Ahora bien, era necesario un replanteamiento de la estrategia: «esta batalla no puede ganarse con un ciego egoísmo profesional o con un empleo desafortunado del derecho de petición o de iniciativa». Con esta consideración, Navarro reconocía dos cuestiones claves que habían lastrado seriamente la campaña anterior. La primera, la estrecha vinculación a las reivindicaciones corporativas de los catedráticos de instituto; la segunda, los riesgos inherentes a las campañas de peticiones, aspecto que se tratará en el último apartado de este artículo. En los siguientes años la lucha tendría que operar en otro terreno y para ello era necesaria la unidad paciente en un «frente reformista». Porque la batalla por la reforma del bachillerato era, a juicio de Navarro, «una auténtica batalla política». 
El SEPEM se aprestó a librar esta batalla cuando en 1951 el nuevo ministro Joaquín Ruiz-Giménez anunció su intención de reformar la Ley de 1938. Fernández-Pacheco remitió varias circulares a directores e inspectores en los que solicitaba de nuevo la redacción de escritos y la remisión de telegramas al Caudillo, al ministro y a los procuradores. ${ }^{\text {xi }}$ Se reeditaba, por tanto, el modelo de campaña de 1946-47.

Tras una tensa negociación con la Iglesia, la ley de 1953 derogó finalmente el modelo instaurado en 1938 y suprimió el polémico examen de Estado, sustituyéndolo por reválidas al final de los nuevos bachilleratos elemental y superior. Ahora bien, resulta difícil afirmar que la Iglesia fuera privada de derechos o prerrogativas sustanciales en el nuevo bachillerato. Por el contrario, la nueva ley supuso el revés definitivo para las reivindicaciones que vehiculaba el SEPEM. El examen de Estado desaparecía, pero los catedráticos eran sustituidos en los tribunales de las nuevas reválidas de cuarto y sexto por inspectores, incluso en los que examinaban a los alumnos oficiales. Además, los institutos perdían toda competencia inspectora en favor de una inspección central, mientras la Iglesia conseguía su propia inspección y, además, seguía fiscalizando las condiciones morales de los centros públicos. El único logro que podían esgrimir los falangistas era la paternidad del añadido del artículo primero que establecía que «el Estado procurará que esta Enseñanza, al menos en su grado elemental, llegue a todos los españoles». Pero incluso esta vaga promesa de futura extensión se acompañaba de la pérdida del monopolio tradicional de la enseñanza oficial sobre los sectores menos favorecidos a causa del reconocimiento de la función social de la enseñanza privada religiosa. El último escrito de catedráticos impulsado por el SEPEM daba cuenta de esta derrota. En 1953 lo único que los catedráticos pedían ya era que les dejaran como mínimo examinar a sus propios alumnos. ${ }^{\text {xii }} \mathrm{Y}$ ni eso se consiguió.

\section{LA DEBILIDAD ESTRUCTURAL DEL SEPEM}

Los sucesivos fracasos de las campañas impulsadas por el SEPEM determinaron su práctica desaparición tras la aprobación de la ley de 1953. Más allá de la correlación de fuerzas y de la habilidad de sus dirigentes, en el origen de este fracaso se encontraba una debilidad estructural. En realidad, el SEPEM nunca dispuso de instrumentos para mantener la fidelidad y la disciplina de aquellos a los que encuadraba, tanto en la enseñanza oficial como en la privada.

Con respecto a la enseñanza pública, el Servicio no tenía competencias disciplinarias sobre el profesorado y una vez superada la fase de la depura- 
ción y acotado el matonismo falangista, los catedráticos podían eludir las consignas de los falangistas de la Delegación de Educación. El delegado de Baleares emitía ya en 1945 un lúcido diagnóstico sobre esta falta de poder efectivo:

Mientras sea posible que el Director de Instituto o de otro Centro docente cualquiera, tenga el camino libre para resolver las cuestiones profesionales, docentes, etc. sin el visto bueno, consentimiento, o como quiera llamarse de la Delegación Provincial de Educación y pueda decir que, en su actuación, para nada le interesan las Delegaciones de Educación, ni la opinión ni las resoluciones de Falange y que, la realidad nos pueda enseñar realmente que obra al margen de toda actividad de carácter falangista, será efectivamente muy insignificante la labor que las Delegaciones de Educación puedan desarrollar en la enseñanza oficial. ${ }^{\text {xiii }}$

Tampoco disponía el SEPEM de influencia significativa en temas cruciales como el ingreso al cuerpo. Por otro lado, los traslados, otra cuestión fundamental para el profesorado, eran competencia de la sección segunda del Consejo de Educación Nacional en la que la presencia del SEPEM se limitaba a Navarro.

Privado de mecanismos de coacción y sin capacidad para desarrollar una red clientelar propia, el SEPEM se veía condenado a pugnar por convertirse en canal de expresión de las insatisfacciones de sus encuadrados para mantener su fidelidad. Pero esta estrategia contenía potenciales riesgos para su supervivencia que el Servicio no pudo conjurar.

El primer riesgo de esta estrategia era el de ser instrumentalizado por, o vincularse excesivamente, a un sector profesional concreto, como sucedió con los catedráticos de instituto. Como se ha expuesto, la supeditación a sus reivindicaciones corporativas determinó no sólo las posiciones del Servicio en el debate sobre el bachillerato de los años cuarenta, sino también el abanico de cuestiones planteadas. No se detecta en el entorno falangista preocupación alguna por temas centrales en el debate educativo europeo de entreguerras como la apertura social de la enseñanza media o la integración orgánica de la constelación de otros estudios medios en una estructura común, cuestiones en las que sus homólogos alemanes e italianos presionaron frente al carácter conservador de las primeras políticas educativas fascista y nazi. Significativamente, las únicas reflexiones en este sentido fueron planteadas tempranamente por el primer responsable de la enseñanza media, José Pemartín, quien no encontró eco ni en católicos ni en falangistas. 
Quizás el ejemplo más revelador de esta subordinación a los intereses corporativos de los catedráticos de instituto sea el de la formación política. El adoctrinamiento de la juventud en los valores falangistas, así como la educación premilitar, luego física, fue asignado a un profesorado especial nombrado por el Frente de Juventudes o la Sección Femenina. Este profesorado especial fue fuente de tensiones permanentes en los centros. Más allá de la afinidad ideológica, su condición mayoritaria de no licenciados y la naturaleza de las enseñanzas que impartían los convertía en elementos ajenos a un profesorado en el que estaban muy presentes los valores académicos y de cuerpo. A grandes rasgos, sus materias se devaluaron y cualquier intento de trascender este marco chocaba con la complicidad del resto del profesorado que les privaba de autoridad ante los alumnos. Las quejas en este sentido por parte de este profesorado eran constantes. Así, por ejemplo, ya en 1943 ante las protestas de las instructoras de la Sección Femenina del instituto de Santiago por la indisciplina generalizada de las alumnas consentida, a su juicio, por la directora, esta respondía a la delegada de la Sección poniendo en cuestión la moralidad de las instructoras. ${ }^{\text {xxiv }}$

Los informes de los delegados provinciales de educación, mayoritariamente licenciados y catedráticos de instituto, cuestionaban abiertamente la capacidad docente de estos instructores, su preparación intelectual e incluso su honradez. Presidía estos informes un cierto desdén hacia estos elementos ajenos que se habían colocado gracias al favor político. La solución a esta escasa credibilidad de la formación política pasaba por su adscripción a los catedráticos. El propio SEPEM asumió esta propuesta en 1945, lo que le llevó a subvertir la lógica fascista alemana e italiana y a sufrir una nueva derrota, esta vez frente a secciones del propio partido, pues tanto la Sección Femenina como el Frente de Juventudes se negaron con éxito a ceder sus competencias sobre la educación política.

El segundo reto que la estrategia del SEPEM debía superar era el mantenimiento de la unidad de acción y la disciplina de los movilizados para evitar que sus intervenciones fuesen más allá de lo deseado. Este fue el caso del claustro del instituto de Huesca que, en la última fase de la movilización de 1946-47, redactó una carta en la que cuestionaba abiertamente la utilidad del recurso a la jerarquía católica y reclamaba una mayor decisión al Ministerio. ${ }^{x v}$ Y lo más significativo; se negó a rectificar pese al disgusto de Navarro y las presiones de los dirigentes locales del SEPEM. xxvi

Finalmente, el escollo definitivo que el SEPEM no pudo superar en la enseñanza pública fue el de ofrecer algún tipo de satisfacción, aunque fuera parcial, a los movilizados. Tras la esterilidad de la movilización de los años cuarenta, el fracaso de 1953 y la incapacidad para controlar la formación 
política en los centros poco sentido tenía seguir confiando en el Servicio siquiera por motivos instrumentales.

Tampoco consiguió el SEPEM retener competencias que permitieran consolidar su ascendencia sobre el profesorado de los colegios privados. Ciertamente, a través de los Colegios Oficiales de Licenciados y Doctores había conseguido erigirse desde mediados de los años cuarenta en representante de este sector en el ámbito laboral. La presión del Servicio consiguió que se publicaran las bases de trabajo de 1946 e incluso que se suspendiera la posibilidad de despidos. Sin embargo, esta derivación sindical se vio pronto bloqueada. En 1951, la intervención de la Iglesia forzó al Ministerio de Trabajo a reivindicar para la organización sindical las competencias sobre condiciones laborales que el SEPEM se había atribuido en la práctica y frustró todos los planes del Servicio en este sentido. ${ }^{\text {xvii }}$ Con ello, todo el andamiaje institucional levantado por el SEPEM quedaba limitado a la defensa de unos intereses profesionales, nunca laborales, que, como reconocía el jefe del SEPEM de Valladolid, además no eran homogéneos para todo el colectivo. ${ }^{\text {xviii }}$

Desprovisto de poder efectivo sobre cuestiones claves como el acceso al cuerpo, la promoción, el régimen disciplinario o las condiciones laborales de sus encuadrados e incapaz de proveer algún tipo de satisfacción a sus reivindicaciones, no resulta extraño que a lo largo de los cincuenta el Servicio languideciera como una pieza más del enorme engranaje burocrático del Movimiento.

\section{CONCLUSIONES}

Desde 1942, los falangistas encontraron en el SEPEM el organismo desde el que desafiar la hegemonía alcanzada por los católicos en el bachillerato a través del encuadramiento y movilización del profesorado tanto público como privado. En el seno del organismo se definió un programa de reforma del bachillerato que cuestionaba los pilares del modelo definido en 1938 y a lo largo de los años cuarenta el SEPEM mantuvo abierto el debate sobre el bachillerato dirigiendo una notable movilización del profesorado en favor de su reforma.

Sin embargo, la actuación del Servicio resultó un fracaso. Desprovisto de mecanismos efectivos de control y coacción sobre el profesorado, la estrategia del SEPEM se basó en erigirse en el canal de expresión de las reivindicaciones de aquellos a los que encuadraba. Pero esta era una estrategia arriesgada, pues obligaba al Servicio a ofrecer satisfacción en algún grado a 
las insatisfacciones del profesorado para retener su adhesión. El mundo educativo católico movió las piezas para evitar que esto ocurriera. En el caso del profesorado privado, los católicos encontraron aliados en el propio Movimiento que privaron a los Colegios Oficiales de Licenciados y Doctores de toda competencia laboral. Más difícil resultó conjurar el desafío planteado por la movilización del profesorado público, pero la Iglesia consiguió finalmente salir indemne del intento de reforma impulsado por el propio Ministerio en 1947. Ni siquiera pudo el Servicio obtener réditos de la reforma de 1953, pues apenas ninguna de sus reivindicaciones fue atendida en el nuevo modelo.

El mismo SEPEM fue en gran medida responsable de este fracaso final, pues su voluntad de satisfacer a los catedráticos de institutos agraviados por la ley de 1938 le convirtió en rehén de sus reivindicaciones corporativas. El falangismo renunció a elaborar su propio proyecto educativo y a introducir en el debate cuestiones de mayor alcance que sus homólogos italianos y alemanes no habían dudado en plantear. Posiblemente, una organización de profesorado no era la más adecuada para hacerlo, pero en todo caso la estrecha vinculación a las reivindicaciones del cuerpo de catedráticos resultó letal para la reforma defendida por el Servicio. Y es que, en la medida en que esas reivindicaciones atentaban frontalmente contra la autonomía alcanzada por los colegios religiosos, la batalla planteada por el SEPEM resultaba una batalla perdida de antemano bajo el primer franquismo. 


\section{NOTAS}

I. Consúltense las estadísticas elaboradas por el Instituto de la Mujer -sección «Mujer en Cifras»-(http://www.inmujer.migualdad.es/mujer/), el INE (www.ine.es) y el Ministerio de Educación (http://www.educacion.es/portada.html).

II. Circular reorganizando el Servicio de Educación Nacional, Boletín del Movimiento, 1 de junio de 1940 y Bases orgánicas de la Delegación Nacional de Educación, 12 de noviembre de 1942. Presidencia, Caja 52/14155, Archivo General de la Administración (en adelante, AGA).

III. Información Universitaria, 52, (1941) y 53 (1941).

IV. Información Universitaria, 100, (1942).

V. Información Universitaria, 102, (1942).

VI. Información Universitaria, 112, (1942), p. 322.

VII. La semana se convocó inicialmente para la última semana de septiembre, aunque posteriormente se retrasó hasta la segunda semana de octubre y finalmente se celebro en diciembre. Información Universitaria, 113, (1942) y 115 (1942).

VIII. Orden de 14 de agosto de 1942. BOE de 22 de agosto de 1942.

IX. (1943). Primera semana de enseñanza media oficial. Revista Nacional de Educación, 25, y (1942) de Enseñanza Media. Información Universitaria, 129.

X. (1950). Isaac Peral, 2, 1 izqda. Zaragoza. Educación y Cultura, 51-52.

XI. Colegio Oficial de Doctores y Licenciados del distrito Universitario de Madrid, Resumen de la estadística sobre el funcionamiento de los Centros reconocidos del D.U. de Madrid, en el curso 1942-43, 19 de octubre de 1943. Educación, Caja 31/6022, AGA.

XII. (1945). Acuerdos que ha adoptado la Comisión permanente del S.E.P.E.M. Información Universitaria, 226, pp. 54-57.

XIII. (1945). Sustancioso mensaje. Información Universitaria, 228.

XiV. La cursiva es mía.

XV. (1946). Los graduados y la Segunda Enseñanza. Información Universitaria, 285.

XVI. Respectivamente en Información Universitaria, 303, (1946), 304 (1946), 307 (1946), 308309 (1946), 311 (1946) y 313 (1946).

XVII. José M. Gallart (1946). Hablemos claro. Información Universitaria, 311.

XVIII. (1946). Sobre el examen de Estado. Información Universitaria, 303.

XIX. Información Universitaria, 304 (1946).

XX. Antonio Fernández Pacheco, Circular reservada, s.f. Presidencia, Caja 14168, AGA.

XXI. Escrito a la Jerarquía Eclesiástica sobre los problemas actuales de la Enseñanza Media, 12 de octubre de 1947. Presidencia, Caja 1457, AGA.

XXII. Navarro Latorre, J., Circular n. 106. La reforma general de la enseñanza media española, 13 de julio de 1949. Presidencia, Caja 14156, AGA.

XXIII. Para una síntesis de la estrategia de la campaña, véase Fernández-Pacheco, A. Nota Circular, 27 de noviembre de 1951. Presidencia, Caja 14156, AGA.

XXIV. Los catedráticos de instituto dicen, 1953, Presidencia, Caja 19035, AGA.

XXV. Memoria interesada en circular 30 de diciembre, 11 de abril de 1945, Presidencia, Caja 14154, AGA. 
XXVI. [Informe de la Delegada de la Sección Femenina de Santiago], 27 de agosto de 1943. Presidencia, Caja 18974, AGA.

XXVII. [Carta de profesores del instituto Ramón y Cajal de Huesca a José Navarro Latorre], 22 de octubre de 1947. Presidencia, Caja 14168, AGA.

XXVIII. Informe sobre el catedrático Ramón Martín Blesa, 4 de diciembre de 1947. Presidencia, Caja 14160, AGA.

XXIX. Nota a la propuesta de organismo profesional para la reglamentación laboral docente presentada al misterio de Trabajo por secretariado de la Comisión Episcopal de la Enseñanza, mayo de 1951, Presidencia, Caja 14156, AGA y J. Navarrro Latorre, Los problemas del S.E.P.E.M., 29 de enero de 1952, Presidencia, Caja 14156, AGA.

XXX. Arribas, F. Los colegios oficiales, su misión y desenvolvimiento, 21 de enero de 1951. Presidencia, Caja 14159, AGA. 


\section{REFERENCIAS BIBLIOGRAFICAS}

Alcoba López, A. (2002). Auge y ocaso de El Frente de Juventudes. Madrid: San Martín.

Alfonso Sánchez, J. M. (1999). Iglesia y Estado. La actuación de la Iglesia católica en la legislación educativa, 19381953. Madrid: Fundación Universitaria Española.

Alted Vigil, A. (1984). Política del Nuevo Estado sobre el patrimonio cultural y la educación durante la Guerra Civil española. Madrid: Ministerio de Cultura.

Cámara Villar, G. (1984). Nacional-catolicismo y escuela: la socialización politica del franquismo, 1936-1951. Jaén: Hesperia.

Cruz Orozco, J. I. (2001). El yunque azul. Frente de Juventudes y sistema educativo: razones de un fracaso. Madrid: Alianza.

Delgado García, L. (2004). Política educativa y orientación escolar y profesional en España entre 1939 y 1968. Cuenca: Alfonsópolis.

Galfré, M. (2000). Una riforma alla prova. La scuola media de Gentile e il fascismo. Milán: Franco Agnelli.

González Gallego, I. (1988). Falange y educación. Zaragoza, 1936-1940. Historia de la Educación, 7, 203-230.

Lorenzo Vicente, J. A. (2003). La enseñanza media en la España franquista, 1936-1975. Madrid: Editorial Complutense.

Morente Valero, F. (2001). «Libro e Moschetto". Politica educativa y politica de juventud en la Italia fascista (19221943). Barcelona: PPU.

Morente Valero, F. (2005), Los fascismos europeos y la política educativa del franquismo. Historia de la Educación, 24, 179-204.

Noval Clemente, M. (1999). La Sección Femenina en Murcia: educación, cultura e ideología (1939-1977). Murcia: Universidad de Murcia. (Tesis doctoral)

Ruiz Carnicer, M. A. (1996). El Sindicato Español Universitario (SEU), 19391965. Madrid: Siglo XXI.

Ruiz Rico, J. J. (1977). El papel político de la Iglesia católica en la España de Franco (1936-1971). Madrid: Tecnos.

Sáez Marín, J. (1988). El Frente de Juventudes. Política de juventud en la España de la postguerra (1937-1960). Madrid: Siglo XXI. 


\section{PALABRAS CLAVE}

Falange, franquismo, Iglesia, política de la educación, enseñanza secundaria, profesión docente.

\section{KEYWORDS}

Falange, Francoism, Catholic Church, educational policy, secondary education, teaching personnel.

\section{PERFIL ACADEMICO DEL AUTOR}

Antonio Fco. Canales Serrano es doctor en Historia por la Universidad Autónoma de Barcelona y profesor en Facultad de Educación de la Universidad de La Laguna. Ha centrado su trayectoria investigadora en el franquismo, periodo sobre el que ha publicado diversos artículos y el libro Las otras derechas (Madrid, Marcial Pons, 2006). Actualmente investiga sobre la educación y la ciencia en la posguerra. Ha editado recientemente, junto a Amparo Gómez, el libro Ciencia y fascismos. La ciencia española de posguerra (Barcelona, Laertes, 2009).

Dirección del Autor: Antonio Fco. Canales Serrano

Facultad de Educación

Universidad de La Laguna

Avda. Trinidad, s.n.

38204 La Laguna

S.C. Tenerife

Telf: $922254089-657667168$

acanales@ull.es

Fecha Recepción del Artículo: 10. Junio. 2010

Fecha Revisión del Artículo: 20. Octubre. 2010

Fecha Aceptación del Artículo: 30.Octubre. 2010

Fecha de Revisión para publicación: 04. Julio. 2011 
